\title{
Magnetosonic resonance in a dipole-like magnetosphere
}

\author{
A. S. Leonovich ${ }^{1}$, D. A. Kozlov ${ }^{1}$, and V. A. Pilipenko ${ }^{2}$ \\ ${ }^{1}$ Institute of Solar-Terrestrial Physics (ISTP), Russian Academy of Sciences, Siberian Branch, Irkutsk, Russia \\ ${ }^{2}$ Institute of the Physics of the Earth, Russian Academy of Sciences, Moscow, Russia
}

Received: 17 February 2006 - Revised: 4 July 2006 - Accepted: 17 July 2006 - Published: 13 September 2006

\begin{abstract}
A theory of resonant conversion of fast magnetosonic (FMS) waves into slow magnetosonic (SMS) oscillations in a magnetosphere with dipole-like magnetic field has been constructed. Monochromatic FMS waves are shown to drive standing (along magnetic field lines) SMS oscillations, narrowly localized across magnetic shells. The longitudinal and transverse structures, as well as spectrum of resonant SMS waves are determined. Frequencies of fundamental harmonics of standing SMS waves lie in the range of 0.1$1 \mathrm{mHz}$, and are about two orders of magnitude lower than frequencies of similar Alfvén field line resonance harmonics. This difference makes an effective interaction between these MHD modes impossible. The amplitude of SMS oscillations rapidly decreases along the field lines from the magnetospheric equator towards the ionosphere. In this context, magnetospheric SMS oscillations cannot be observed on the ground, and the ionosphere does not play any role either in their generation or dissipation. The theory developed can be used to interpret the occurrence of compressional Pc5 waves in a quiet magnetosphere with a weak ring current.
\end{abstract}

Keywords. Ionosphere (Wave propagation) - Magnetospheric physics (Magnetosphere-ionosphere interactions; MHD waves and instabilities)

\section{Introduction}

In studies of magnetospheric MHD oscillations, one of the most productive ideas was the theory of field line resonance. Having appeared for the first time in Tamao (1965), this theory was later developed by Chen and Hasegawa (1974), Radoski (1974), and Southwood (1974). This theory describes resonant driving of Alfvén field line oscillations by a monochromatic fast magnetosonic (FMS, or compressional)

Correspondence to: A. S. Leonovich

(leon@iszf.irk.ru) wave. The interaction between these modes is confined in a narrow range of magnetic shells, where a local frequency of Alfvén eigenoscillations is close to FMS wave frequency.

The field line resonance theory was first developed for a one-dimensional inhomogeneous model of the magnetosphere, where the magnetic field lines are assumed to be straight, and plasma inhomogeneity is directed across the field lines. This theory was later advanced for magnetosphere models with plasma inhomogeneous, both along magnetic field lines and across magnetic shells with either straight magnetic field lines (Kivelson and Southwood, 1986; Southwood and Kivelson, 1986) or a dipole-like magnetic field (Lifshitz and Fedorov, 1986; Leonovich and Mazur 1989; Chen and Cowley, 1989; Wright, 1992).

In a plasma with finite pressure there is another branch of MHD oscillations - the slow magnetosonic (SMS) mode. SMS waves are in many aspects similar to Alfvén waves: both modes are guided by magnetic field lines and have continuous spectra (Lifshitz and Fedorov, 1986). Many authors (e.g. Southwood, 1977; Walker, 1987; Taylor and Walker, 1987; Walker and Pekrides, 1996) examined the coupling of a small-scale (large azimuthal wave numbers, $m \gg 1$ ) Alfvén mode with SMS mode in a finite-pressure plasma embedded in curvilinear magnetic field. These theories were aimed at understanding the physics of compressional Pc5 waves persistently observed by space-borne magnetometers (Barfield and McPherron, 1978; Woch et al., 1990). Various kinds of instabilities of ring current ions were suggested as a possible excitation mechanism for these waves (Pokhotelov et al., 1986; Cheng and Lin, 1987; Cheng et al., 1994). These theories are relevant to the storm-time periods, when the intensity of the ring current is high enough and effective magnetosonic velocity of energetic particles is about the Alfvén velocity of background plasma. However, some compressional Pc5 waves were observed under very weak ring current $\left(D_{s t} \sim 0\right)$ (Greene et al., 2005), so probably they are excited by another, still unidentified, mechanism. Those Pc5 oscillations

Published by Copernicus GmbH on behalf of the European Geosciences Union. 


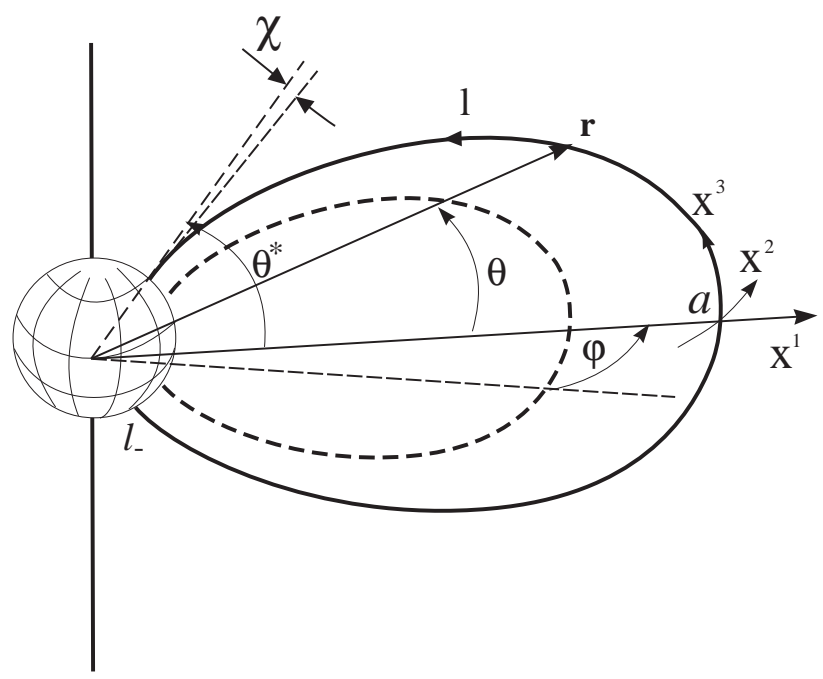

Fig. 1. The curvilinear orthogonal coordinate system $\left(x^{1}, x^{2}, x^{3}\right)$ of the magnetic field lines and the non-orthogonal coordinate system $(a, \phi, \theta)$ used in numerical calculations.

cannot be merely monochromatic FMS waves, because for such waves the magnetosphere is an opacity region, and their amplitude should decrease exponentially upon penetration into the inner magnetosphere at a much smaller scale than its typical size. Klimushkin (1997) suggested that ionospheric currents could be a source of magnetospheric SMS waves. However, as will be shown in this paper, the efficiency of such a generation mechanism is doubtful.

The similarity between the Alfvén and SMS modes suggests that SMS oscillations could be generated through a mode conversion similar to the Alfvén field line resonance, as was demonstrated by Yumoto (1985) within a onedimensional plasma model. The processes of resonant conversion of FMS disturbances into Alfvén or SMS oscillations may be termed the Alfvén and magnetosonic resonances, respectively. During the magnetosonic resonance, monochromatic FMS waves excite SMS oscillations at those magnetic shells where the FMS wave frequency coincides with the local frequency of SMS eigenoscillations.

In this paper we develop the theory of magnetosonic resonance in a two-dimensional inhomogeneous magnetosphere model with dipole-like magnetic field. Distributions of the main plasma parameters in this model correspond to the mildly disturbed dayside magnetosphere of the Earth. Under these conditions, the typical frequencies of Alfvén and SMS oscillations differ by about two orders of magnitude, so interaction between these modes is very weak, in contrast to the models of the ring current plasma (e.g. Walker, 1987; Taylor and Walker, 1987; Walker and Pekrides, 1996).

The paper is organized as follows. In Sect. 2, we introduce a model of the medium and the basic equations describing the magnetosonic resonance. In Sect. 3, the ionospheric boundary conditions are inferred for the resonant SMS os- cillations. In Sects. 4 and 5, we find solutions in the WKB approximation to equations describing the structure of resonant SMS oscillations along and across the magnetic field lines. In Sect. 6, analytical equations are obtained representing the components of the field of resonant SMS waves in the resonant region. In Sect. 7, we solve numerically the equations describing the structure and spectrum of three first harmonics of resonant standing SMS oscillations, and compare the spatial structures of Alfvén and magnetosonic resonances. Finally, in the Discussion and Conclusion sections, we summarize the principal results and discuss possible verification of the proposed theory.

\section{The model and the basic equations}

We consider coupled MHD oscillations in a magnetosphere model with a dipole-like magnetic field (Fig. 1). We introduce a curvilinear orthogonal coordinate system $\left(x^{1}, x^{2}, x^{3}\right)$ of the magnetic field lines, in which the coordinate $x^{3}$ is oriented along the field line, $x^{1}$ is directed across the magnetic shells, and the azimuthal $x^{2}$ coordinate completes the righthand coordinate system. The squared length element in this coordinate system is determined as

$d s^{2}=g_{1}\left(d x^{1}\right)^{2}+g_{2}\left(d x^{2}\right)^{2}+g_{3}\left(d x^{3}\right)^{2}$,

where $g_{i}(i=1,2,3)$ are metric coefficients. We assume that the plasma and magnetic field are homogeneous along the azimuthal coordinate $x^{2}$. We employ the system of MHD equations for an ideal plasma with a finite isotropic pressure:

$\rho \frac{d \mathbf{v}}{d t}=-\nabla P+\frac{1}{4 \pi}[\operatorname{curl} \mathbf{B} \times \mathbf{B}]$,

$\frac{\partial \mathbf{B}}{\partial t}=\operatorname{curl}[\mathbf{v} \times \mathbf{B}]$,

$\frac{\partial \rho}{\partial t}+\nabla(\rho \mathbf{v})=0$,

$\frac{d}{d t} \frac{P}{\rho^{\gamma}}=0$,

where $\mathbf{B}$ and $\mathbf{v}$ are the magnetic field and plasma velocity, $\rho$ and $P$ are the plasma density and pressure, and $\gamma$ is the adiabatic index.

Application of the ideal MHD approximation is well justified for oscillations in collisional plasma. The magnetospheric plasma is more likely to be collisionless for the oscillations under study. To fully examine the oscillations in such a medium, one should use the kinetic theory. However, the kinetic theory of MHD oscillations in two- and threedimensional inhomogeneous plasma is too complex and does not always allow for rigorous solutions of the resulting equations. For our purpose - to examine the structure of MHD oscillations in an inhomogeneous medium - the MHD approximation is usually applied, with some reservations. The substantiation of its applicability in this case is the CGL (ChewGoldberg-Low) theory for oscillations in magnetized plasma (Chew et al., 1956). 
To determine the oscillatory electric field $\mathbf{E}$ we use the frozen-in condition

$\mathbf{E}=-\frac{[\mathbf{v} \times \mathbf{B}]}{c}$.

In a stationary condition $(\partial / \partial t=0)$, the system $(1-4)$ describes the distribution of the parameters of an unperturbed magnetosphere: $\mathbf{B}_{\mathbf{0}}, \mathbf{v}_{\mathbf{0}}, \mathbf{E}_{\mathbf{0}}, \rho_{0}$, and $P_{0}$. We assume that the background plasma flow is absent, so $\mathbf{v}_{\mathbf{0}}=0$, and $\mathbf{E}_{\mathbf{0}}=0$. Let us linearize the system (1-4) with respect to small perturbations as follows: $\mathbf{B}=\mathbf{B}_{\mathbf{0}}+\tilde{\mathbf{B}}, \quad \mathbf{v}=\tilde{\mathbf{v}}, \quad \mathbf{E}=\tilde{\mathbf{E}}, \quad \rho=\rho_{0}+\tilde{\rho}$, and $P=P_{0}+\tilde{P}$, where the $\tilde{\mathbf{B}}, \tilde{\mathbf{v}}, \tilde{\mathbf{E}}, \tilde{\rho}$, and $\tilde{P}$ are related to MHD oscillations. Each of the perturbed components can be represented as harmonics of the Fourier expansion $\propto \exp \left(i k_{2} x^{2}-i \omega t\right)$, where $\omega$ is the oscillation frequency, and $k_{2}$ is the azimuthal wave number (if $x^{2}=\phi$ is the azimuth angle, then $\left.k_{2}=m=1,2,3 \ldots\right)$. From Eq. (1) we have

$-i \omega \rho_{0} v_{1}=-\nabla_{1} \widetilde{P}+\frac{B_{0}}{4 \pi} \frac{1}{\sqrt{g_{3}}}\left(\nabla_{3} B_{1}-\nabla_{1} B_{3}\right)$,

$-i \omega \rho_{0} v_{2}=-i k_{2} \widetilde{P}-\frac{B_{0}}{4 \pi} \frac{1}{\sqrt{g_{3}}}\left(i k_{2} B_{3}-\nabla_{3} B_{2}\right)$,

$i \omega \rho_{0} v_{3}=\nabla_{3} \widetilde{P}$,

where $v_{i}$, and $B_{i}(i=1,2,3)$ are the covariant components of the perturbed velocity $\tilde{\mathbf{v}}$ and magnetic field $\tilde{\mathbf{B}}$, and $\nabla_{i} \equiv \partial / \partial x^{i}$. From Eqs. (3), (4) we obtain

$$
\begin{aligned}
\widetilde{P}=-i \frac{\gamma}{\omega} \frac{P_{0}^{1-\sigma}}{\sqrt{g}}\left[\nabla_{1}\left(\frac{\sqrt{g}}{g_{1}} P_{0}^{\sigma} v_{1}\right)+\right. \\
\left.\quad i k_{2} \frac{\sqrt{g}}{g_{2}} P_{0}^{\sigma} v_{2}+\nabla_{3}\left(\frac{\sqrt{g}}{g_{3}} P_{0}^{\sigma} v_{3}\right)\right],
\end{aligned}
$$

where $g=\sqrt{g_{1} g_{2} g_{3}}$, and $\sigma=1 / \gamma$.

It is convenient to describe the MHD field components $\tilde{\mathbf{B}}$, $\tilde{\mathbf{v}}, \tilde{\mathbf{E}}, \tilde{\rho}$, and $\tilde{P}$ via electromagnetic potentials. According to the Helmholtz expansion theorem (Korn and Korn, 1968), an arbitrary vector field, at any point of which its first derivative is determined, can be represented as a sum of potential and vortex fields. For the two-dimensional vector $\tilde{\mathbf{E}}=\left(E_{1}, E_{2}, 0\right)$ this expansion has the form

$\tilde{\mathbf{E}}=-\nabla_{\perp} \varphi+\left[\nabla_{\perp}, \Psi\right]$,

where $\nabla_{\perp} \equiv\left(\nabla_{1}, \nabla_{2}\right)$ is the transverse 2 -dimensional Laplacian, $\varphi$ and $\Psi$ are the scalar and vector potentials, respectively. Under proper gauge calibration, the vector potential has only a longitudinal (field-aligned) component, $\Psi=\left(0,0, \psi_{3} \equiv \psi\right)$. Using the linearized system (1-4) we express the perturbed field components through the potentials $\varphi$ and $\psi$ as

$$
\begin{aligned}
& E_{1}=-\nabla_{1} \varphi+i k_{2} \psi, \quad E_{2}=-i k_{2} \varphi-\nabla_{1} \psi, \quad E_{3}=0, \\
& B_{1}=\frac{c}{\omega} \frac{g_{1}}{\sqrt{g}} \nabla_{3}\left(k_{2} \varphi-i \frac{g_{2}}{\sqrt{g}} \nabla_{1} \psi\right),
\end{aligned}
$$

$$
\begin{aligned}
& B_{2}=\frac{c}{\omega} \frac{g_{2}}{\sqrt{g}} \nabla_{3}\left(i \nabla_{1} \varphi+k_{2} \frac{g_{1}}{\sqrt{g}} \psi\right), \\
& B_{3}=i \frac{c}{\omega} \frac{g_{3}}{\sqrt{g}}\left(\nabla_{1} \frac{g_{2}}{\sqrt{g}} \nabla_{1} \psi-k_{2}^{2} \frac{g_{1}}{\sqrt{g}} \psi\right), \\
& v_{1}=-\frac{c p^{-1}}{B_{0}}\left(i k_{2} \varphi+\frac{g_{2}}{\sqrt{g}} \nabla_{1} \psi\right), \\
& v_{2}=\frac{c p}{B_{0}}\left(\nabla_{1} \varphi-i k_{2} \frac{g_{1}}{\sqrt{g}} \psi\right), \quad v_{3}=-i \frac{\nabla_{3} \widetilde{P}}{\omega \rho_{0}} .
\end{aligned}
$$

The perturbed pressure is described by the following equation

$$
\begin{aligned}
\widehat{L}_{0} \widetilde{P}=i \gamma \frac{c}{\omega} \frac{P_{0}^{1-\sigma}}{\sqrt{g}} & {\left[i k_{2} \varphi \nabla_{1} \frac{\sqrt{g_{3}} P_{0}^{\sigma}}{B_{0}}+\right.} \\
& \left.\nabla_{1} \frac{p P_{0}^{\sigma}}{B_{0}} \nabla_{1} \psi-k_{2}^{2} \frac{p^{-1} P_{0}^{\sigma}}{B_{0}} \psi\right],
\end{aligned}
$$

where $p=\sqrt{g_{2} / g_{1}}$, and the operator $\widehat{L}_{0}$ is

$\widehat{L}_{0}=\frac{\gamma}{\omega^{2}} \frac{P_{0}^{1-\sigma}}{\sqrt{g}} \nabla_{3} \frac{\sqrt{g}}{g_{3}} \frac{P_{0}^{\sigma}}{\rho_{0}} \nabla_{3}+1$.

Let us multiply Eq. (5) by $i k_{2} B_{0} / \rho_{0}$, and Eq. (6) by $B_{0} / \rho_{0}$, then differentiate the latter with respect to $x^{1}$, and subtract the obtained equations one from another. As a result we obtain

$$
\begin{aligned}
\nabla_{1} \widehat{L}_{T} \nabla_{1} \varphi & -k_{2}^{2}\left(\widehat{L}_{P} \varphi+\right. \\
& \left.\frac{S^{2}}{A^{2}} \frac{\varphi}{\sqrt{g_{1} g_{2}}} \nabla_{1} \ln B_{0} \nabla_{1} \ln \frac{\sqrt{g_{3}} P_{0}^{\sigma}}{B_{0}}\right)= \\
& i \frac{k_{2}}{\omega}\left(\nabla_{1} \widehat{L}_{T} \frac{g_{1}}{\sqrt{g}} \psi-\widehat{L}_{P} \frac{g_{2}}{\sqrt{g}} \nabla_{1} \psi\right) .
\end{aligned}
$$

Here $S$ and $A$ denote characteristic plasma velocities: $S=\sqrt{\gamma P_{0} / \rho_{0}}$ is the sound velocity, and $A=B_{0} / \sqrt{4 \pi \rho_{0}}$ is the Alfvén velocity. We have introduced in Eq. (12) the toroidal $\widehat{L}_{T}$ and poloidal $\widehat{L}_{P}$ longitudinal operators, as follows

$$
\begin{aligned}
& \widehat{L}_{T}=\frac{1}{\sqrt{g_{3}}} \nabla_{3} \frac{p}{\sqrt{g_{3}}} \nabla_{3}+p \frac{\omega^{2}}{A^{2}}, \\
& \widehat{L}_{P}=\frac{1}{\sqrt{g_{3}}} \nabla_{3} \frac{p^{-1}}{\sqrt{g_{3}}} \nabla_{3}+p^{-1} \frac{\omega^{2}}{A^{2}} .
\end{aligned}
$$

Now we derive an equation for the magnetosonic mode characterized by the potential $\psi$. For this purpose we apply the operator $\widehat{L}_{0}$ to Eq. (6) and substitute in the obtained equation the components of perturbed field via potentials from Eq. (8). After some rearranging of the terms we have

$$
\begin{aligned}
& \frac{B_{0} \sqrt{g_{3}}}{4 \pi \rho_{0}} \widehat{L}_{0} \frac{B_{0}}{\sqrt{g_{3}}} \widetilde{\Delta} \psi+S^{2} \bar{\Delta} \psi+\omega^{2} \psi= \\
& -i \frac{B_{0} \sqrt{g_{3}}}{4 \pi k_{2} \rho_{0}} \widehat{L}_{0} B_{0} \widehat{L}_{T} \nabla_{1} \varphi-i \varphi k_{2} S^{2} \frac{g_{3}}{\sqrt{g}} \nabla_{1} \ln \frac{\sqrt{g_{3}} P_{0}^{\sigma}}{B_{0}},
\end{aligned}
$$




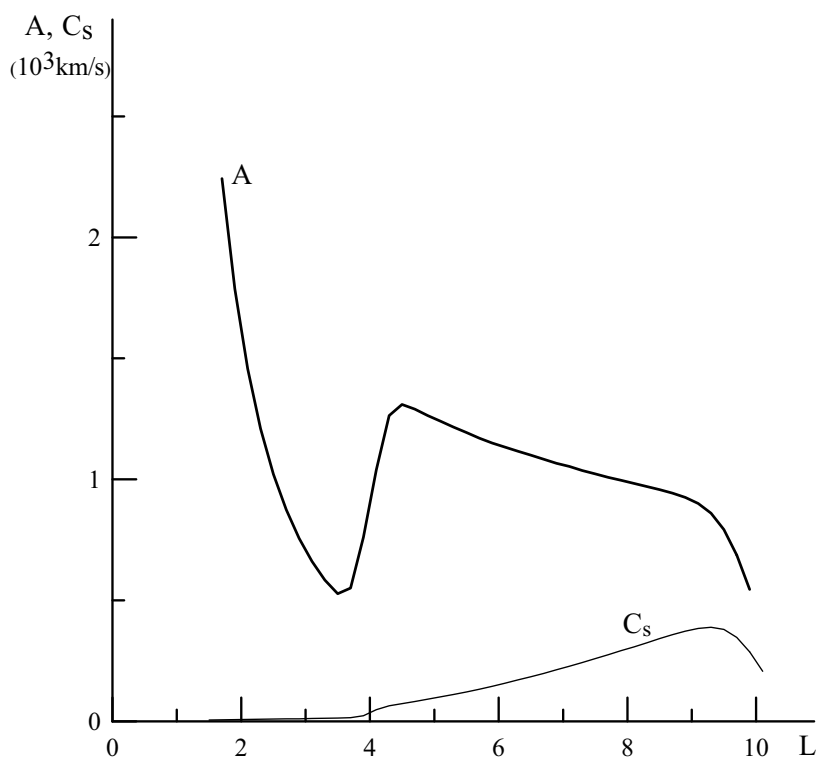

Fig. 2. Equatorial distribution of the Alfvén velocity $A$ and the velocity of SMS waves $C_{S}$ across the magnetic shells $\left(L=a / R_{E}\right.$, $R_{E}$ is the Earth's radius).

where $\widetilde{\Delta}$ and $\bar{\Delta}$ are the differential operators analogous to the Laplace operator:

$$
\begin{gathered}
\widetilde{\Delta}=\frac{g_{3}}{\sqrt{g}} \nabla_{1} \frac{g_{2}}{\sqrt{g}} \nabla_{1}-\frac{k_{2}^{2}}{g_{2}}+\nabla_{3} \frac{g_{2}}{\sqrt{g}} \nabla_{3} \frac{g_{1}}{\sqrt{g}}, \\
\bar{\Delta}=\frac{B_{0}}{P_{0}^{\sigma}} \frac{1}{\sqrt{g_{1} g_{2}}}\left(\nabla_{1} \frac{p P_{0}^{\sigma}}{B_{0}} \nabla_{1}-\right. \\
\left.\frac{k_{2}^{2}}{p} \frac{P_{0}^{\sigma}}{B_{0}}+\nabla_{3} \frac{\sqrt{g}}{g_{3}} \frac{P_{0}^{\sigma}}{\rho_{0}} \nabla_{3} \frac{\rho_{0}}{B_{0} \sqrt{g_{3}}}\right) .
\end{gathered}
$$

Equations (12) and (13) form a closed system for the potentials $\varphi$ and $\psi$. In a homogeneous plasma, the right-hand parts of these equations vanish. Then, the operator in the left-hand part of (12) provides the dispersion equation for the Alfvén waves $\omega^{2}=k_{\|}^{2} A^{2}$, where $k_{\|}^{2} \equiv k_{3}^{2} / g_{3}$ is the field-aligned component of the wave vector. The operator in the left-hand part of Eq. (13) yields the dispersion equation for the slow and fast magnetosonic waves:

$\omega^{4}-\omega^{2} k^{2}\left(A^{2}+S^{2}\right)+k^{2} k_{\|}^{2} A^{2} S^{2}=0$,

where $k^{2}=k_{\|}^{2}+k_{\perp}^{2}$ is the squared total wave vector, and $k_{\perp}^{2}=k_{1}^{2} / g_{1}+k_{2}^{2} / g_{2}$ is the squared transverse wave vector component. Thus, Alfvén oscillations are described by the scalar potential $\varphi$, and magnetosonic modes are characterized by the longitudinal component $\psi$ of the vector potential. In an inhomogeneous plasma, the right-hand parts of Eqs. (12) and (13) describe the interaction of Alfvén and magnetosonic modes. The solution of the dispersion equation (14) can be represented as

$\omega^{2}=\frac{k^{2}}{2}\left(A^{2}+S^{2}\right) \pm \sqrt{\frac{k^{4}}{4}\left(A^{2}+S^{2}\right)^{2}-k^{2} k_{\|}^{2} A^{2} S^{2}}$.

Here the plus/minus sign corresponds to the FMS/SMS waves. If one of the inequalities $S \ll A, A \ll S$, or $\left|k_{\|}\right| \ll\left|k_{\perp}\right|$ holds, the following approximate dispersion equations can be obtained:

$\omega^{2} \approx k^{2} C_{F}^{2}$ for the FMS waves, where $C_{F}^{2}=A^{2}+S^{2}$ and $\omega^{2} \approx k_{\|}^{2} C_{S}^{2}$ for the SMS waves, where $C_{S}^{2}=A^{2} S^{2} /\left(A^{2}+S^{2}\right)$.

FMS oscillations can freely propagate in the magnetosphere within their transparency regions. As for SMS oscillations, which, in magnetized plasma, are a modification of the ion-sound wave branch, some doubts exist whether they can exist in the magnetosphere as an eigenmode. It is known (see Krall and Trivelpiece, 1973) that in plasma with a very anisotropic distribution of ion and electron temperatures (at $T_{e} \gg T_{i}$ ), the decrement of these oscillations due to their interaction with the background plasma ions is sufficiently small $\left(\operatorname{Im} \omega / \operatorname{Re} \omega \sim \sqrt{m_{e} / m_{i}} \ll 1\right)$. In this case, a branch of almost undamped SMS oscillations can well exist. However, such conditions are rare enough in the Earth's magnetosphere and can only be realised in the region of maximum concentration of high-energy electrons in the outer radiation belt, in the regions of intensive parallel currents, or in the morning sector of the magnetosphere at the substorm periods. In other regions of the magnetosphere, the anisotropy of electron and ion temperatures is more likely to be inverse $\left(T_{i} \gtrsim T_{e}\right)$. In this case the decrement of SMS oscillations under study is not small. Estimates show that in homogeneous plasma $\operatorname{Im} \omega / \operatorname{Re} \omega \sim 0.1$, and such oscillations are difficult to be regarded as an eigenmode. Hopefully, inclusion of plasma inhomogeneity will decrease the decrement due to the interaction of the waves with background ions. This is caused by deteriorating conditions for resonant interaction between the waves and particles along geomagnetic field lines. In this case the SMS oscillations may display themselves as noneigenmode oscillations driven by an external source. For example, as is the case in our problem, the decrement of SMS oscillations may be quite sufficient for them to stand out against the exciting FMS wave.

In the following calculations we use the spatial distribution of the plasma and magnetic field parameters provided by the self-consistent model of the magnetosphere (Leonovich et al., 2004). Distribution of these parameters in the magnetic meridian plane corresponds well to a moderately disturbed dayside magnetosphere. Radial distributions of the Alfvén velocity $A(L, 0)$ (where $L=a / R_{E}$, and $a$ is the equatorial radius of the magnetic shell) and of the SMS wave velocity $C_{S}(L, 0)$ in the equatorial plane, according to this model, are shown in Fig. 2.

At least one of the above mentioned inequalities (e.g. $S \ll A$ ) is valid throughout the entire magnetosphere (with 
the exception of the plasma layer). In this approximation the dispersion equation for SMS waves is very similar to the dispersion equation for Alfvén waves. The velocity of SMS waves, as well as of Alfvén waves, is directed along magnetic field lines. In an inhomogeneous plasma this provides a necessary condition for the resonant excitation of SMS oscillations by monochromatic FMS waves. Although the potential $\psi$ describes both the fast and slow magnetosonic modes, in the linear approximation this potential can be decomposed as the sum of the component $\psi_{F}$, related to the FMS wave, and $\psi_{S}$, related to the SMS wave, that is $\psi=\psi_{F}+\psi_{S}$. It can be shown that in a homogeneous plasma the perturbation of total pressure by SMS waves $\left(\varphi=0, \psi=\psi_{S}\right)$ is

$$
\left(\widetilde{P}+P_{m}\right) / \widetilde{P} \sim\left(\widetilde{P}+P_{m}\right) / P_{m}=\frac{k_{\|}^{2}}{k^{2}} \frac{A^{2}}{A^{2}+S^{2}},
$$

where $P_{m}=B_{0} B_{\|} / 4 \pi$ is the perturbed magnetic pressure. For oscillations with small-scale transverse structure, $k_{\perp} \gg k_{\|}$, it follows from the above that an SMS wave practically does not disturb the total pressure

$\widetilde{P}+P_{m} \approx 0$.

For the magnetosphere the typical eigenfrequencies of fundamental harmonics of standing Alfvén and SMS waves, as determined by the background plasma, differ considerably. This means that the interaction of Alfvén and SMS waves, which may occur in a finite-pressure plasma embedded in a curved magnetic field (Southwood and Saunders, 1985), is negligible. Therefore, while examining the SMS oscillation structure described by Eq. (13), one sets $\varphi=0$ in its righthand part.

As will be seen from the calculations below, the resonant SMS waves are strongly localized across magnetic shells near the resonant shell. Away from it, the main contribution to potential $\psi$ comes almost exclusively from the FMS oscillations $\left(\psi \approx \psi_{F}\right)$. Neglecting the small component $(\sim S / A \ll 1)$ related to the derivatives along the longitudinal coordinate $x^{3}$ in the operator $\widehat{L}_{0}$ in Eq. (13), we obtain an equation that describes the FMS wave field far from the resonant surface:

$A^{2} \widetilde{\Delta} \psi_{F}+S^{2} \bar{\Delta} \psi_{F}+\omega^{2} \psi_{F}=0$

An approximate solution of Eq. (16) was found by Leonovich and Mazur (2000). They showed that the back influence from the resonant mode to the driving FMS wave was small, so the decoupled Eq. (16) can be used to describe FMS oscillations throughout the entire region of their existence, even inside the resonant region.

In the vicinity of the resonant surface the total magnetic field of magnetosonic oscillations is composed from the FMS and SMS wave fields: $\psi=\psi_{F}+\psi_{S}$. Substituting this expression for the potential $\psi$ into Eq. (13) and taking into account
Eq. (16) we obtain the equation for the resonant SMS oscillations:

$$
\begin{aligned}
& \frac{B_{0} \sqrt{g_{3}}}{4 \pi \rho_{0}} \widehat{L}_{0} \frac{B_{0}}{\sqrt{g_{3}}} \widetilde{\Delta} \psi_{S}+S^{2} \bar{\Delta} \psi_{S}+\omega^{2} \psi_{S}= \\
& -\frac{A^{2} S^{2}}{\omega^{2}} \frac{\rho_{0} / \sqrt{g_{1} g_{2}}}{B_{0} P_{0}^{\sigma}} \nabla_{3} \frac{\sqrt{g}}{g_{3}} \frac{P_{0}^{\sigma}}{\rho_{0}} \nabla_{3} \frac{B_{0}}{\sqrt{g_{3}}} \widetilde{\Delta} \psi_{F} .
\end{aligned}
$$

The right-hand part of Eq. (17) represents the driver monochromatic FMS wave field, that will be treated as a function known from the solution of Eq. (16). At the frequencies in question the magnetosphere as a whole is an opacity region for FMS. If we accept that the source of FMS oscillations is outside of or at the boundary of the magnetosphere, their amplitudes decreases exponentially inside the magnetosphere on a scale proportional to $m$. FMS oscillations with $m \gg 1$ virtually do not penetrate into the magnetosphere. Only oscillations with $m \sim 1$ on resonant shells have an amplitude sufficient to drive effectively FMS waves. Therefore, we shall consider oscillations with $m \sim 1$.

\section{The boundary condition for the SMS waves in the ionosphere}

To complete the formulation of the resonant SMS wave structure problem, Eq. (17) should be supplemented by the boundary conditions at some coordinates $x^{1}$ and $x^{3}$. Since we suppose that a desired solution for $\psi_{S}$ is localized close to the resonant surface $\left(x^{1}=x_{\text {res }}^{1}\right)$, a natural boundary condition is that the wave amplitude $\psi_{S}$ should decrease away from $x_{\text {res }}^{1}$.

Another boundary condition is set at the intersection of geomagnetic field lines with the conductive layer of the northern and southern ionospheres. A solution of the problem concerning the structure of the Alfvénic-type wave field in the atmosphere and ionosphere for an inclined geomagnetic field was found by Leonovich and Mazur (1996). Using the same approach, we obtain the following ionospheric boundary condition for the transverse components of the SMS wave electric field

$$
\left.\frac{\partial E_{n, y}}{\partial l}\right|_{l=l_{ \pm}}=-i \frac{\omega}{v_{ \pm}} E_{n, y}\left(l_{ \pm}\right)-i \frac{I_{n, y}\left(l_{ \pm}\right)}{V_{ \pm}} .
$$

Here the signs " \pm " refer to the intersection points of the field lines with the northern and southern ionospheres; $l$ is the coordinate measured along the field line from the equator, $d l=\sqrt{g_{3}} d x^{3}$, and $E_{n}=E_{1} / \sqrt{g_{1}}$ and $E_{y}=E_{2} / \sqrt{g_{2}}$ are the physical components of the wave electric field. The other parameters are the ionospheric Pedersen velocity $v_{ \pm}$ (Leonovich and Mazur, 1991; Pilipenko et al., 2000) and $V_{ \pm}$

$v_{ \pm}=\frac{c^{2} \cos \chi_{ \pm}}{4 \pi \Sigma_{p}^{( \pm)}} ; \quad V_{ \pm}=\frac{\omega}{v_{ \pm} \Sigma_{p}^{( \pm)}}$.

The height-integrated (across the ionospheric conducting layer with thickness $\Delta$ ) Pedersen conductivity and surface 
density of transverse external current $\mathbf{j}^{(e x t)}$ in the ionosphere are

$\Sigma_{p}=\int_{0}^{\Delta} \sigma_{p} d z ; \quad I_{x, y}=\int_{0}^{\Delta} j_{x, y}^{(e x t)} d z$,

where $I_{x}=I_{n} \cos \chi$, and $\chi$ is the angle between the vertical and the field line at the intersection point with the upper boundary of the ionosphere (see Fig. 1). Assuming that in Eq. (8) only the component $\psi_{S}$, related to the SMS oscillations, is non-vanishing, we obtain from Eq. (18):

$$
\left.\psi_{S}\right|_{l_{=l_{ \pm}}}=\left.\mp i \frac{v_{ \pm}}{\omega} \frac{\partial \psi_{S}}{\partial l}\right|_{l=l_{ \pm}}+i \frac{J^{( \pm)}}{\Sigma_{P}^{( \pm)}},
$$

where the functions $J^{( \pm)} \equiv J\left(l=l_{ \pm}\right)$are related to the density of external currents in the ionosphere by

$\Delta_{\perp} J=\int_{0}^{\Delta}\left(\nabla \times \mathbf{j}^{(e x t)}\right)_{z} d z$

where $\Delta_{\perp}=\nabla_{x}^{2}+\nabla_{y}^{2}$ is the transverse Laplacian. The first term in the right-hand part of Eq. (19) describes the decay of SMS waves due to the Joule dissipation in the conducting ionosphere, while the second item describes a possible driving of SMS waves by external currents in the ionosphere.

\section{The field-aligned structure of standing SMS waves}

In the following calculations we will consider the fieldaligned structure of several first harmonics of standing SMS waves. Typical field-aligned wave length of such oscillations is on the order of the field line length. As we will see, the typical scale of resonant SMS oscillations across magnetic shells is much smaller than their longitudinal wave length, $\left|\nabla_{1} \psi_{s} / \psi_{s}\right| \gg\left|\nabla_{3} \psi_{s} / \psi_{s}\right|$. Therefore, a solution to Eq. (17) may be sought using the method of different scales, representing the potential $\psi_{S}$ as

$\psi_{S}=U\left(x^{1}\right)\left(S\left(x^{1}, x^{3}\right)+h\left(x^{1}, x^{3}\right)\right)$,

where the function $U\left(x^{1}\right)$ describes the small-scale transverse structure of oscillations along the coordinate $x^{1}$ in the main order, whereas the function $S\left(x^{1}, x^{3}\right)$ describes the oscillation structure along magnetic field lines. The typical scale of $S\left(x^{1}, x^{3}\right)$ along $x^{1}$ is assumed to be much larger than the scale of $U\left(x^{1}\right)$. The small correction term $h\left(x^{1}, x^{3}\right)$ describes the oscillation structure in higher orders of the perturbation theory.

An equation for the longitudinal structure can be obtained if one retains in Eq. (17) only the main-order terms $\left(\sim \nabla_{1}^{2} \psi_{S}\right)$ of the perturbation theory:

$\frac{\rho_{0} p^{-1}}{B_{0} P_{0}^{\sigma}} \nabla_{3} \frac{\sqrt{g} P_{0}^{\sigma}}{g_{3} \rho_{0}} \nabla_{3} \frac{B_{0}}{g_{1} \sqrt{g_{3}}} S+\frac{\omega^{2}}{C_{S}^{2}} S=0$.
We assume that the functions $S\left(x^{1}, x^{3}\right)$ in the main order satisfy the homogeneous boundary conditions in the ionosphere: $S\left(x^{1}, x_{ \pm}^{3}\right)=0$. The solution of the spectral problem (21) are the eigenfunctions $S_{N}\left(x^{1}, x^{3}\right)(N=1,2,3 \ldots$ is the harmonic number) and the corresponding eigenfrequencies $\omega^{2}=\Omega_{S N}^{2}$. The eigenfunctions are normalized by the following condition

$$
\int_{l_{-}}^{l_{+}} \frac{p P_{0}^{\sigma}}{g_{1} g_{3}} \frac{A^{2}}{C_{S}^{2}} S_{N}^{2} d l=1 .
$$

In order to obtain a qualitative representation of the structure of the eigenfunctions $S_{N}\left(x^{1}, x^{3}\right)$ in question, let us find a solution to Eq. (21) in the WKB approximation, applicable for harmonics with $N \gg 1$. Using the standard method (e.g. Leonovich and Mazur, 1993), one may find the solution of Eq. (21) normalized by the condition (22), in the first two orders of the WKB approximation as follows

$S_{N}\left(x^{1}, x^{3}\right)=\sqrt{\frac{2}{t_{S}} \frac{g_{3} g_{1}^{3 / 2}}{P_{0}^{\sigma} g_{2}^{1 / 2}} \frac{C_{S}}{A^{2}}} \sin \left(\Omega_{S N} \int_{l_{-}}^{l} \frac{d l}{C_{S}}\right)$.

Here $\Omega_{S N}$ is the eigenfrequency determined by the travel time $t_{S}$ of SMS wave between the conjugate ionospheres, namely

$\Omega_{S N}=\frac{2 \pi N}{t_{S}}, \quad t_{S}=\int_{l_{-}}^{l_{+}} \frac{d l}{C_{S}}$.

A solution to Eq. (21) for the fundamental and lower harmonics $(N \sim 1)$ can be found only numerically. The numerical solution of Eq. (21) in the magnetosphere model under consideration is discussed in Sect. 7.

\section{Transverse structure of the resonant SMS-wave}

We assume that there are no external currents in the ionosphere, so the correction term $h_{N}\left(x^{1}, x^{3}\right)$ in Eq. (20) satisfies the following ionospheric boundary condition (see Eq. 19):

$\left.h_{N}\right|_{l=l_{ \pm}}=\left.\mp i U_{N}\left(x^{1}\right) \frac{v_{ \pm}}{\omega} \frac{\partial S_{N}}{\partial l}\right|_{l=l_{ \pm}}$.

Let us pre-multiply Eq. (17) by $S_{N} p P_{0}^{\sigma} / \sqrt{g_{3}} S^{2}$ and integrate along the field line between the conjugate ionospheres. Taking into account (21) and the boundary condition (25), we obtain for the function $U_{N}\left(x^{1}\right)$ the following equation:

$$
\begin{aligned}
& \frac{\left(\omega^{2}+i \gamma_{N}\right)^{2}-\Omega_{S N}^{2}}{\omega^{2}} \nabla_{1}^{2} U_{N}^{2}- \\
& {\left[\beta_{1 N}+\left(k_{2}^{2} \beta_{2 N}+\beta_{3 N}\right) \frac{\left(\omega^{2}+i \gamma_{N}\right)^{2}-\Omega_{S N}^{2}}{\omega^{2}}\right] U_{N}=\Gamma_{N},}
\end{aligned}
$$


where

$$
\begin{aligned}
& \beta_{1 N}=\int_{l_{-}}^{l_{+}} \frac{p P_{0}^{\sigma}}{g_{3}} S_{N}\left(\nabla_{3} \frac{g_{2}}{\sqrt{g}} \nabla_{3} \frac{g_{1}}{\sqrt{g}} S_{N}+\frac{\Omega_{S N}^{2}}{C_{S}^{2}} S_{N}\right) d l, \\
& \beta_{2 N}=\int_{l_{-}}^{l_{+}} \frac{p P_{0}^{\sigma}}{g_{2} g_{3}} \frac{A^{2}}{C_{S}^{2}} S_{N}^{2} d l, \\
& \beta_{3 N}=-\int_{l_{-}}^{l_{+}} \frac{p P_{0}^{\sigma}}{g_{3}} \frac{A^{2}}{S^{2}} S_{N} \nabla_{3} \frac{g_{2}}{\sqrt{g}} \nabla_{3} \frac{g_{1}}{\sqrt{g}} S_{N} d l, \\
& \Gamma_{N}=\frac{\Omega_{S N}^{2}}{\omega^{2}} \int_{l_{-}}^{l_{+}} \frac{p P_{0}^{\sigma}}{g_{3}} \frac{A^{2}}{C_{S}^{2}} S_{N} \widetilde{\Delta} \psi_{F} d l .
\end{aligned}
$$

The damping rate caused by the Joule dissipation of the SMS wave energy in the northern $\left(l=l_{+}\right)$and southern $\left(l=l_{+}\right)$ ionospheres is described by

$$
\begin{aligned}
\gamma_{N}=\frac{1}{2 \Omega_{S N}^{2}}\left[\frac{p P_{0}^{\sigma} A^{2}}{g_{1} g_{3}} v_{+}\right. & \left.\frac{\partial S_{N}}{\partial l}\right|_{l=l_{+}}+ \\
& \left.\left.\frac{p P_{0}^{\sigma} A^{2}}{g_{1} g_{3}} v_{-} \frac{\partial S_{N}}{\partial l}\right|_{l=l_{-}}\right] .
\end{aligned}
$$

The expressions for the coefficients of Eq. (26) become much simpler for oscillations with $N \gg 1$, when the WKB approximation along the longitudinal coordinate is applicable, namely

$\beta_{1 N} \approx 0, \quad \beta_{2 N} \approx \frac{1}{t_{S}} \int_{l_{-}}^{l_{+}} \frac{g_{1}}{g_{2}} \frac{d l}{C_{S}}, \quad \beta_{3 N} \approx \frac{\Omega_{S N}^{2}}{T_{S}} \int_{l_{-}}^{l_{+}} \frac{g_{1} d l}{C_{S} S^{2}}$, $\gamma_{N}=\frac{1}{t_{S}}\left[\frac{v_{+}}{C_{S}^{+}}+\frac{v_{-}}{C_{S}^{-}}\right]$.

In this approximation the damping rate does not depend on the harmonic number $N$ or oscillation frequency of standing SMS waves.

Now we find a solution to Eq. (26) in the vicinity of the resonant magnetic shell $x^{1}=x_{S N}^{1}$, where $\omega=\Omega_{S N}\left(x_{S N}^{1}\right)$. Let the function $\Omega_{S N}\left(x^{1}\right)$ change monotonically, so that in the vicinity of the resonant surface one may approximate $\Omega_{S N}\left(x^{1}\right)$ by a linear dependence

$\Omega_{S N}\left(x^{1}\right) \approx \omega\left(1-\frac{x^{1}-x_{S N}^{1}}{L}\right)$.

This approximation is valid at $\left|x^{1}-x_{S N}^{1}\right| \ll L$, where $L=\left|\partial \ln \Omega_{S N} / \partial x^{1}\right|^{-1}$ is the typical scale of $\Omega_{S N}$ variation at the point $x^{1}=x_{S N}^{1}$. Substituting Eq. (28) into Eq. (26) and introducing the dimensionless variable $\xi=\left(x^{1}-x_{S N}^{1}\right) / \lambda_{S N}$, where $\lambda_{S N}=1 / \sqrt{\beta_{3 N}}$, we obtain an equation describing the transverse structure of magnetosonic resonance

$(\xi+i \varepsilon) \frac{\partial^{2} U_{N}}{\partial \xi^{2}}-\left[c_{N}+\left(1+d_{N}\right)(\xi+i \varepsilon)\right] U_{N}=G_{N}$.

The coefficients of this equation are: $\varepsilon=\gamma_{N} L / \omega \lambda_{S N}$ is the dimensionless width of the resonance, $c_{N}=\beta_{1 N} L \lambda_{S N}$, $d_{N}=\beta_{2 N} k_{2}^{2} \lambda_{S N}^{2}$, and $G_{N}=\Gamma_{N} \lambda_{S N} L$. These coefficients may be considered as constants because they vary insignificantly within the localization region of the desired solution $U_{N}(\xi)$.

Solution to Eq. (29) can be found by representing the desired function $U_{N}(\xi)$ as a Fourier integral

$U_{N}(\xi)=\frac{1}{\sqrt{2 \pi}} \int_{-\infty}^{\infty} \bar{U}_{N}(k) e^{i k \xi} d k$

Substituting this equation into Eq. (29) we obtain a first-order differential equation for the function $\bar{U}_{N}(k)$. This equation can be solved easily (Leonovich and Mazur, 1997), and after the inverse Fourier transformation we obtain a solution to the initial equation in the form

$U_{N}(\xi)=i G_{N}(0) \int_{0}^{\infty} \frac{\exp [i k(\xi+i \varepsilon)+i \zeta(k)]}{k^{2}+1+d_{N}} d k$,

where

$\zeta(k)=\frac{c_{N}}{\sqrt{1+d_{N}}} \arctan \frac{k}{\sqrt{1+d_{N}}}$.

Let us consider the behavior of $U_{N}(\xi)$ in the region near the resonant plane $(\xi \rightarrow 0)$ and at large asymptotic distances $(|\xi| \rightarrow \infty)$. As $\xi \rightarrow 0$ the bulk of the integrand (30) accumulates in the domain $k \gg 1$, so that one may set $\zeta(k) \approx \zeta(\infty)$ in the exponent, and neglect all the terms but $k^{2}$ in the denominator. Thus, we obtain an expression for the second derivative

$U_{N}^{\prime \prime}(\xi) \stackrel{\xi \rightarrow 0}{\approx} \frac{G_{N}(0) e^{i \zeta(\infty)}}{(\xi+i \varepsilon)}$

After integration of this expression we obtain

$U_{N}(\xi) \stackrel{\xi \rightarrow 0}{\approx} G_{N}(0) e^{i \zeta(\infty)}(\xi+i \varepsilon) \ln (\xi+i \varepsilon)$.

Such asymptotic behavior of $U_{N}(\xi)$ at $\xi \rightarrow 0$ can also be obtained from Eq. (29) by the Frobenius method.

In the asymptotic $|\xi| \rightarrow \infty$ the bulk of the integrand (30) accumulates in the domain $k \ll 1$, so that one may set $k=0$ in $\zeta(k)$ and in the denominator. After that the integral is easily calculated

$U_{N}(\xi) \stackrel{|\xi| \rightarrow \infty}{\approx}-\frac{G_{N}(0)}{1+d_{N}} \frac{1}{\xi+i \varepsilon}$.

Thus, the amplitude of the resonant SMS oscillations away from the resonant plane decreases asymptotically as $\propto|\xi|^{-1}$. Such behavior satisfies the boundary conditions on the coordinate $x^{1}$. 


\section{Spatial structure of the SMS wave components in the resonant region}

Let us consider the behavior of various components of the field of resonant SMS oscillations close to the resonant shell, $|\xi| \rightarrow 0$. For the magnetic field components we use the expressions (9) in which we set $\varphi=0$, and $\psi=\psi_{S N}$. Using the relationship (31) as an approximate representation of the function $U_{N}(\xi)$, we obtain

$$
\begin{aligned}
& B_{1 N} \approx-i \frac{\bar{B}_{N}}{\lambda_{S N}} \ln (\xi+i \varepsilon)\left(\frac{g_{1}}{\sqrt{g}} \nabla_{3} \frac{g_{2}}{\sqrt{g}} S_{N}\right), \\
& B_{2 N} \approx k_{2} \bar{B}_{N}(\xi+i \varepsilon) \ln (\xi+i \varepsilon)\left(\frac{g_{2}}{\sqrt{g}} \nabla_{3} \frac{g_{1}}{\sqrt{g}} S_{N}\right), \\
& B_{3 N} \approx i \frac{\bar{B}_{N}}{\lambda_{S N}^{2}} \frac{1}{(\xi+i \varepsilon)} \frac{S_{N}}{g_{1}},
\end{aligned}
$$

where $\bar{B}_{N}=(c / \omega) G_{N}(0) e^{i \zeta(\infty)}$. The longitudinal (compressional) magnetic component $B_{3 N}$ has the strongest singularity, $\propto \xi^{-1}$. The radial magnetic component $B_{1 N}$ has a weaker logarithmic singularity, but it dominates close to the ionosphere, where the component $B_{3 N} \propto S_{N}\left(x^{1}, x^{3}\right)$ has a node. The azimuthal component $B_{2 N}$ is regular.

For the electric field components we use Eqs. (8). Substituting into them $U_{N}$ in the form of Eq. (31) we obtain

$E_{1 N} \approx i k_{2} \bar{E}_{N}(\xi+i \varepsilon) \ln (\xi+i \varepsilon)\left(\frac{g_{1}}{\sqrt{g}} S_{N}\right)$,

$E_{2 N} \approx-\frac{\bar{E}_{N}}{\lambda_{S N}} \ln (\xi+i \varepsilon)\left(\frac{g_{2}}{\sqrt{g}} S_{N}\right)$,

where $\bar{E}_{N}=G_{N}(0) e^{i \zeta(\infty)}$. The azimuthal electric component $E_{2 N}$ has a weak logarithmic singularity as $\xi \rightarrow 0$, whereas the radial component $E_{2 N}$ is finite at the resonant point.

For the velocity components, the relationships (10), as well as Eq. (11) relating the perturbed velocity to the perturbed pressure, give us

$$
\begin{aligned}
& v_{1 N} \approx-\frac{\bar{v}_{N}}{\lambda_{S N}} \ln (\xi+i \varepsilon) \frac{S_{N}}{\sqrt{g_{3}}}, \\
& v_{2 N} \approx-i k_{2} \bar{v}_{N}(\xi+i \varepsilon) \ln (\xi+i \varepsilon) \frac{S_{N}}{\sqrt{g_{3}}}, \\
& v_{3 N} \approx-\frac{\bar{v}_{N} / \rho_{0}}{\lambda_{S N}^{2} \omega^{2}} \frac{1}{(\xi+i \varepsilon)} \nabla_{3} \frac{A^{2} \rho_{0}}{g_{1}} \frac{S_{N}}{\sqrt{g_{3}}},
\end{aligned}
$$

where $\bar{v}_{N}=\left(c / B_{0}\right) G_{N}(0) e^{i \zeta(\infty)}$. The spatial structure of the oscillatory plasma velocity is somewhat different from the electromagnetic field structure. Both transverse components $v_{1 N}$ and $v_{2 N}$ have a node close to the ionosphere. Thus, the longitudinal field-aligned component $v_{3 N}$, having a singularity $\propto \xi^{-1}$, dominates throughout an entire resonant region.

The expression for the perturbed plasma pressure near the resonant plane has the form

$$
\widetilde{P}_{N} \approx-i \frac{\bar{B}_{N} B_{0}}{4 \pi \lambda_{S N}^{2}} \frac{1}{(\xi+i \varepsilon)} \frac{S_{N}}{g_{1} \sqrt{g_{3}}} .
$$

Thus, the oscillatory plasma pressure, like the magnetic field compression $B_{3 N}$, has a singular character in the resonant region. As expected, the pressure oscillations are out of phase with the oscillations of the magnetic field compression. It is easy to verify that for the field components of the resonant SMS wave the condition (15) of vanishing total pressure is met.

\section{Results of numerical calculations}

Only numerical solutions can be found to Eqs. (21) and (29), describing the spatial structure of magnetosonic resonance, for the fundamental and low- $N$ harmonics. For a numerical solution we use the coordinate system $(a, \phi, \theta)$ related to the dipole magnetic field lines (Fig. 1): $a$ is the equatorial radius of the field line, $\phi$ is the azimuthal angle, and $\theta$ is the latitude measured from the equator. In this coordinate system the length of the radius vector to a field line point $(r, \theta)$ is $r=a \cos ^{2} \theta$, and the length element along this field line is $d l=a \cos \theta \sqrt{1-3 \sin ^{2} \theta}$. The dipole magnetic field magnitude is

$B_{0}(a, \theta)=B_{0}\left(\frac{a_{0}}{a}\right)^{3} \frac{\sqrt{1-3 \sin ^{2} \theta}}{\cos ^{6} \theta}$,

and the metric tensor components are

$$
g_{1}=\frac{\cos ^{6} \theta}{1-3 \sin ^{2} \theta}, \quad g_{2}=a^{2} \cos ^{6} \theta .
$$

However, there is no similar simple relationship for the component $g_{3}$. It can be determined through the ratio of the segments of field lines with equatorial radii $a_{0}$ and $a$ crossed by two nearby coordinate planes $x^{3}=$ const at latitudes $\theta_{0}$ and $\theta$, respectively:

$$
\frac{g_{3}(a, \theta)}{g_{3}\left(a_{0}, \theta_{0}\right)}=\left(\frac{a}{a_{0}}\right)^{6}\left(\frac{\cos \theta}{\cos \theta_{0}}\right)^{12} \frac{1-3 \sin ^{2} \theta_{0}}{1-3 \sin ^{2} \theta} .
$$

The plasma distribution is specified using the self-consistent model of the dipole magnetosphere (Leonovich et al., 2004). Radial distributions of the Alfvén and magnetosonic velocities in the equatorial magnetospheric plane derived from this model are shown in Fig. 2. All the following calculations have been made for the magnetic shell corresponding to the geosynchronous orbit, $a=6.6 R_{E}$.

Figure $3 \mathrm{a}$ shows the radial distributions of the eigenfrequencies of the first three harmonics of standing SMS waves, obtained from the numerical solution of Eq. (21) with the homogeneous boundary conditions at the ionosphere. For comparison, Fig. 3b shows similar distributions of the first three harmonics of standing Alfvén waves for the same model of the magnetosphere. Each figure lists the travel time along the field line between the conjugate ionospheres for the SMS wave $\left(t_{S}\right)$ and Alfvén wave $\left(t_{A}\right)$. These travel times determine the oscillation eigenfrequencies in the WKB approximation. In contrast to the Alfvén waves, the frequencies 

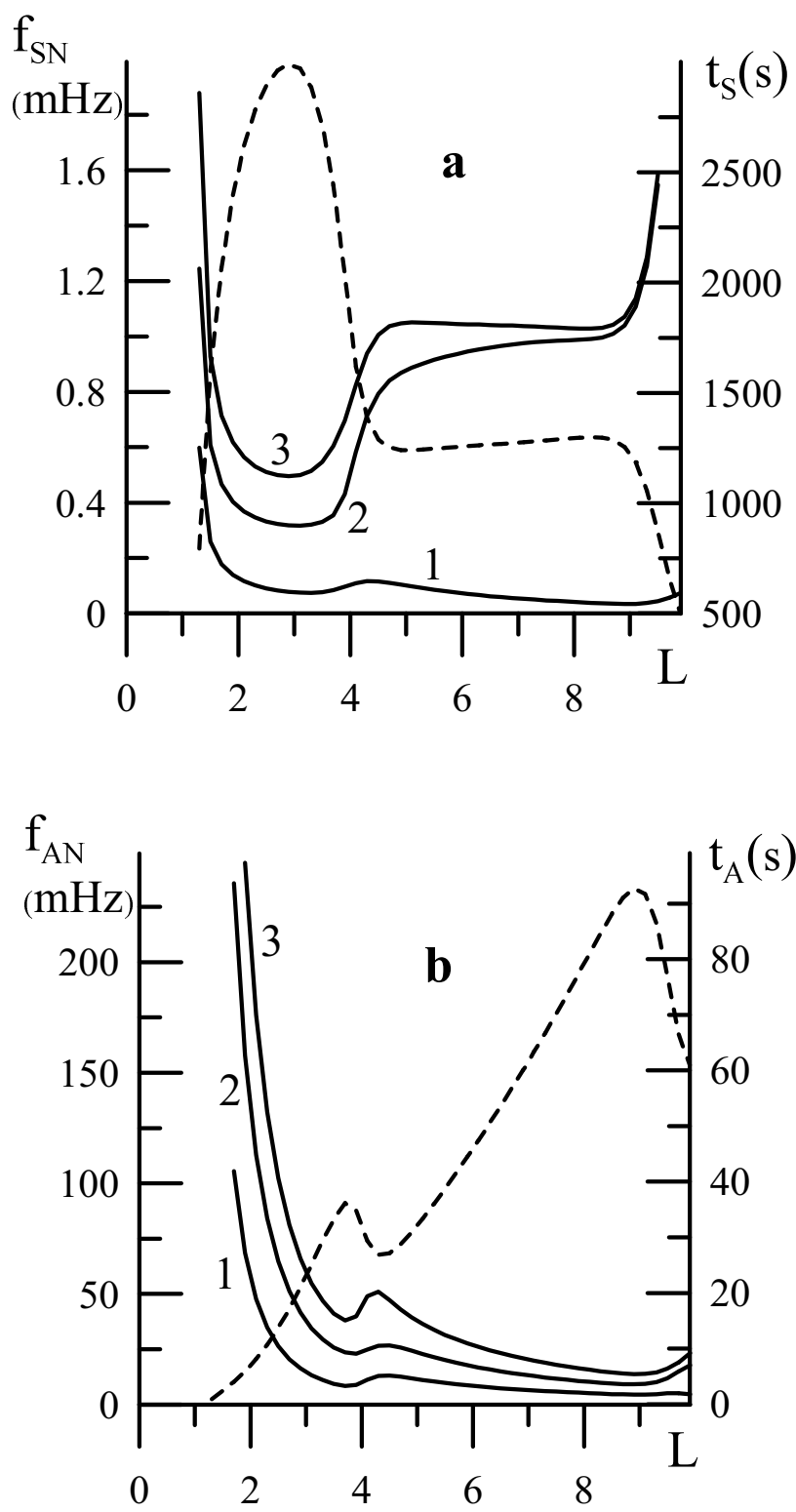

Fig. 3. (a) Distribution across the magnetic shells of eigenfrequencies $f_{S N}$ of the first three harmonics of standing SMS waves and the travel time along the field line between conjugate ionospheres $t_{S}$ with the SMS wave velocity $C_{S}$. (b) Same, for a standing Alfvén wave.

of fundamental harmonics of SMS waves differ greatly from their values in the WKB approximation. For example, the difference between the exact eigenfrequencies of the first SMS harmonic and their WKB estimates is nearly 5 -fold.

Additionally, we would like to pay special attention to the dramatic difference, about two orders of magnitude, between the eigenfrequencies of Alfvén and SMS fundamental harmonics. We can show that the longitudinal structure and the spectrum of SMS oscillations with $m \gg 1$ are determined by an equation analogous to Eq. (21) which is obtained from

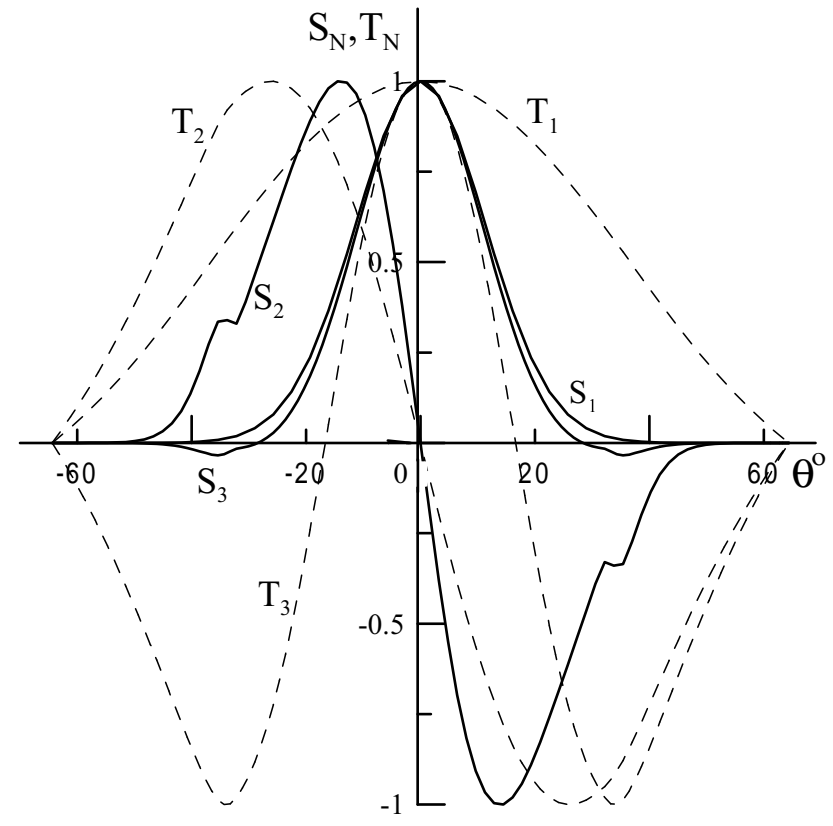

Fig. 4. Structure along the magnetic field lines of the first three harmonics of standing Alfvén waves $T_{N}(\theta)(N=1,2,3)$ (dashed lines) and SMS waves $S_{N}(\theta)(N=1,2,3)$ (solid lines) at the magnetic shell $a=6.6 R_{E}$. Both functions are normalized to unity.

Eq. (17) in the limit $k_{2} \rightarrow \infty$. The spectrum of such oscillations practically coincides with the spectrum of oscillations with $m \sim 1$. This means that an effective interaction between the Alfvén and SMS oscillations is impossible in the magnetosphere which is mildly disturbed by the ring current. This interaction can only be effective in a plasma where $t_{S} \simeq t_{A}$, which may occur in the magnetospheric regions populated with the hot plasma sheet or ring current particles.

Figure 4 shows the field-aligned structures of the first three harmonics of standing Alfvén and SMS waves. Both for the resonant Alfvén and for the SMS waves, the solution can be represented in the form $\propto V_{N}\left(x^{1}\right) T_{N}\left(x^{1}, x^{3}\right), \quad N=1,2,3$, where the function $V_{N}\left(x^{1}\right)$ describes the small-scale structure of oscillations across magnetic shells, while the function $T_{N}\left(x^{1}, x^{3}\right)$ describes their field-aligned structure. We apply here the solution of the Alfvén resonance problem in the model magnetosphere under consideration from Leonovich (2001). One can see from the figure how very different the longitudinal structures of the Alfvén and SMS waves are. For the Alfvén waves the actual field-aligned structure resembles the expected WKB approximation picture. The fundamental harmonics of standing SMS waves differ from their WKB representation (23) radically.

A small blip in the $S_{N}\left(x^{1}, x^{3}\right)$ curve at $\theta \approx \pm 28^{\circ}$ is related to the peculiarity of the employed magnetospheric model, in which a double plasmapause occurs. In the vicinity of $\theta \approx \pm 28^{\circ}$ the magnetic field line crosses the second plasmapause which does not coincide with the magnetic shell. 

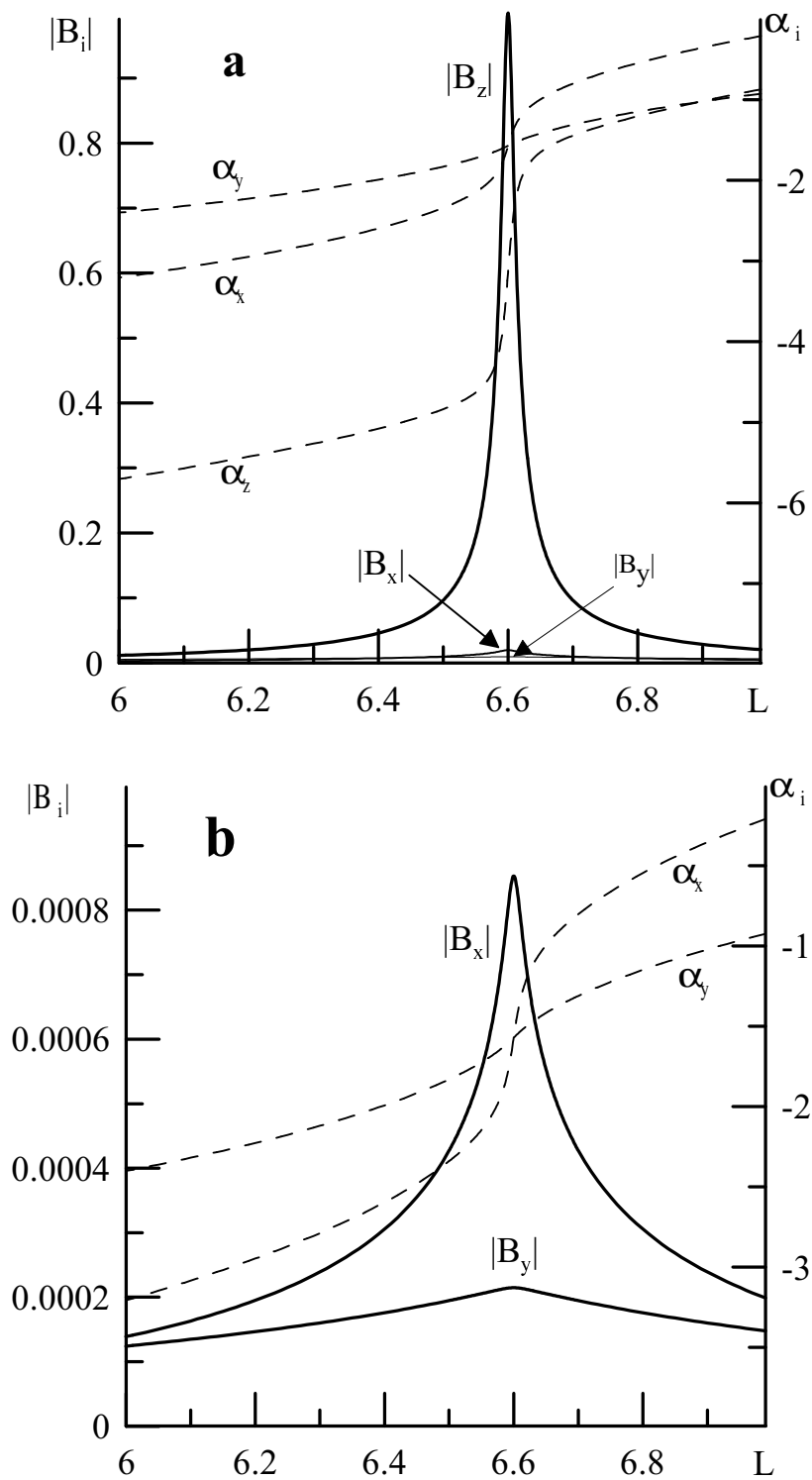

Fig. 5. Transverse structure of magnetic field components $(i=x, y, z)$ of the first harmonic $(N=1)$ of the SMS oscillations in the resonant region near $L=6.6$ : (a) the amplitude $\left|B_{i}\right|$ and phase $\alpha_{i}$ distributions close to the equatorial plane, and (b) above the ionosphere. At the ionospheric boundary the compressional component $B_{z}$ vanishes.

The main peculiarity of fundamental SMS harmonics is the fast decrease of their amplitude upon approaching the ionosphere. Such a structure of $S_{N}\left(x^{1}, x^{3}\right)$ results in a number of important consequences. First, resonant SMS oscillations are impossible to detect on the ground or by a low-orbit satellite. Second, the ionosphere cannot play any significant role either in the generation or energy absorption of magnetospheric SMS waves. This statement follows, for example, from the form of the expression for the damping rate of SMS waves in the ionosphere. From Eq. (27) it follows that $\gamma_{N} \sim\left(\partial S_{N} / \partial l\right)_{l=l_{ \pm}} \rightarrow 0$. An analogous result can also be ob- tained for the external currents in the ionosphere (see Eq. 19), as a possible driver of SMS oscillations. Thus, the ionosphere can neither be a source nor an absorber of the SMS wave energy. SMS wave damping in the magnetosphere must be caused by other mechanisms, probably by their resonant interaction with the the background plasma particles.

Figure 5 shows the radial amplitude-phase structure of the physical components of the wave magnetic field $\left(B_{x} \equiv B_{1} / \sqrt{g_{1}}=\left|B_{x}\right| e^{i \alpha_{x}}, B_{y} \equiv B_{2} / \sqrt{g_{2}}=\left|B_{y}\right| e^{i \alpha_{y}}\right.$, and $\left.B_{z} \equiv B_{3} / \sqrt{g_{3}}=\left|B_{z}\right| e^{i \alpha_{z}}\right)$ of the fundamental harmonic $(N=1)$ of SMS waves near the resonant magnetic shell $a=6.6 R_{E}$. Figure $5 \mathrm{a}$ shows the oscillation structure in the nearequatorial region (far from the nodes of the functions $S_{N}$ and $\partial S_{N} / \partial l$ ). The response shown to the FMS pumping wave is normalized in such a way as to make the peak value at the resonance $\left|B_{z}\right|=1$. The initial oscillation phase is chosen to be zero in an asymptotically distant region to the right of the resonant shell. For numerical calculations the damping rate and imaginary correction factor were chosen to be rather small, $\varepsilon=10^{-2}$, to make the resonant structure evident. The magnitude of the resonant enhancement of SMS oscillations is controlled by the magnitude of the FMS pumping wave and damping rate. When $\gamma_{N}$ and $\varepsilon$ increase, the amplitude maximum decreases and the resonant peak widens. Passing across the resonant peak the phase of the compressional $B_{z}$ component changes approximately by $\pi$, the phase of the $B_{x}$ component by $\sim \pi / 2$, while the phase of the $B_{y}$ component remains practically the same.

Figure $5 \mathrm{~b}$ shows the radial distributions of transverse $\left|B_{x}\right|$ and $\left|B_{y}\right|$ components of the oscillation field close to the ionosphere. It should be remembered that the $\left|B_{z}\right|$ component vanishes in the ionosphere, so that immediately above the ionosphere the horizontal magnetic components prevail over the compressional component. However, because of the fast decrease in the function $\partial S_{N} / \partial l$ when approaching the ionosphere (Fig. 4), the amplitudes of transverse magnetic components are very small close to the ionosphere as compared to their equatorial values.

For comparison, Fig. 6 shows similar calculations for the magnetic components of resonant Alfvén waves. Unlike SMS oscillations, the amplitude of Alfvén oscillations increases (approximately two-fold) from the equator to the ionosphere, owing to the convergence of dipole field lines. The difference in field-aligned structure of the Alfvén and magnetosonic waves may be qualitatively explained as follows. According to the WKB solution (23), the SMS wave amplitude varies as $\propto S_{N}\left(x^{1}, x^{3}\right) \propto A^{-1}$, whereas an analogous function for the Alfvén waves $\propto T_{N}\left(x^{1}, x^{3}\right) \propto \sqrt{A}$. The Alfvén velocity $A$ increases by two orders of magnitude from the equator to the ionosphere. This results in the increase of the Alfvén wave amplitude and a fast decrease of the SMS wave amplitude. Some weaker contributions to the amplitude change are provided by variations of other parameters - the metric tensor components and background plasma pressure. 
For low-frequency FMS waves the Earth is located in the opacity region. Therefore, only large-scale FMS oscillations can reach the ionosphere, though attenuated, but with detectable amplitude. Part of the FMS wave energy is resonantly converted into Alfvén or SMS oscillations. The SMS waves are localized near the equatorial plane of the magnetosphere, so that the Alfvén waves are the only component of MHD oscillations which can reach the Earth's surface.

\section{Discussion}

Satellite observations of intense long-period ULF waves show an antiphase relationship between the magnetic field compression and plasma pressure variations. This relationship is a persistent feature of storm-time Pc5 waves (Barfield and McPherron, 1978; Woch et al., 1990), Pg pulsations (Glassmeier et al., 1999), nightside Pi2-like waves (Nakamizo and Iijima, 2003), etc. This kind of diamagnetic behavior is specific to the SMS mode, thus indicating its involvement in the observed wave structure.

Numerous theories aimed at interpreting the physical mechanism of Pc4-5 waves with dominant compressional magnetic component were based on the notion of Alfvén oscillations coupled with the SMS mode and modified by drift effects and excited by resonant interaction with ring current protons. These theories fairly reasonably explained the observational data, so there was a feeling that the theory of compressional Pc4-5 wave generation was nearly complete (see review by Pilipenko, 1990). However, Takahashi et al. (1987) found a "global Pc5" event where the field-aligned wave scale was much smaller than the Alfvén wavelength. The unique combination of several near-geostationary satellites during the 14-15 November 1979 event gave the possibility to determine the field-aligned structure of exceptionally monochromatic and long-lasting $(\sim 44 \mathrm{~h})$, long-period Pc5-6 oscillations. Rather surprisingly, the field-aligned wave scale (distance from top of field line to wave node) was about $5^{\circ}$ only, much less than the expected Alfvén wave length. Further, Woch et al. (1988) showed that the drastic changes in the compressional Pc5 wave structure during a slight shift of the GEOS-2 satellite from the top of the field line could only be interpreted by invoking a small fieldaligned wave scale. These observations revealed that in the magnetosphere there is at least one sub-class of long-period waves for which all the previously well-elaborated theories of Alfvén mode excitation are not valid. However, these observations still remain the only evidence of the possible occurrence of very small field-aligned structure of long-period ULF waves.

The difficulties with the interpretation of compressional Pc4-5 waves with a short field-aligned scale may be resolved by invoking models based on the excitation of SMS modes. The SMS oscillations can be spontaneously generated during the drift instability of ring current ions with steep radial gra-
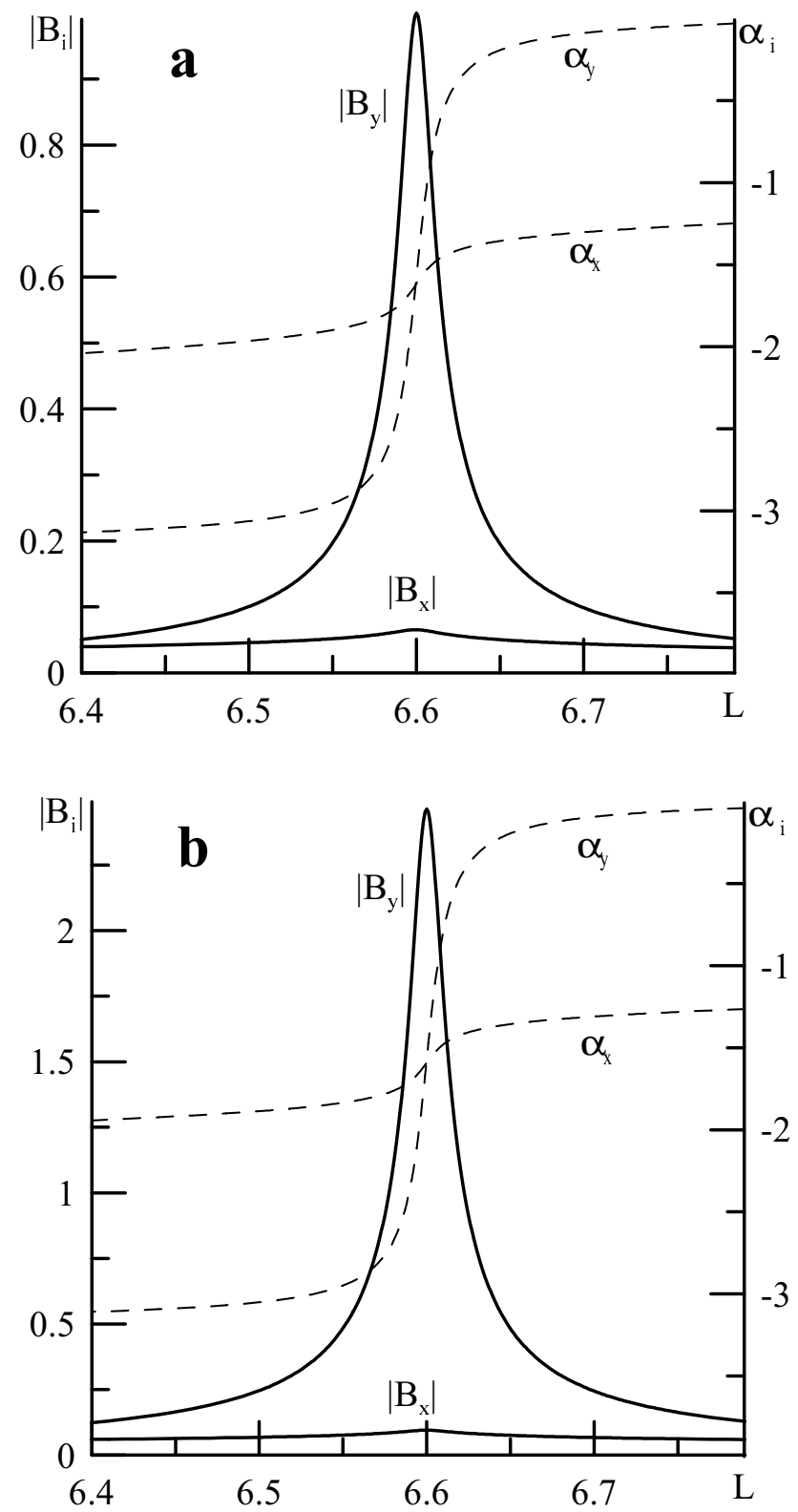

Fig. 6. Transverse structure of magnetic components of the fundamental $(N=1)$ Alfvén oscillations in the region of "classical" Alfvén field line resonance at $L=6.6$ (compare with Fig. 5): the distribution of the wave amplitude $\left|B_{i}\right|$ and phase $\alpha_{i},(i=x, y)$ close to the equatorial plane (a) and above the ionosphere (b).

dients. The field-aligned structure of an unstable SMS mode was shown by Woch et al. (1988) to be much smaller than a field line length.

Our theory predicts that there might be an additional source of compressional Pc4-5 oscillations - the resonant conversion of FMS waves. This mechanism can be most efficient in a mildly disturbed magnetosphere, when fluxes of ring current ions are too low for excitation of instabilities. The mechanism of the drift or bump-on-tail instabilities 
dominates during the main and recovery phases of magnetic storms, when a sufficient amount of the ring current particles have been injected into the inner magnetosphere. The slow velocity of SMS mode results in the severe damping of the SMS mode in an isothermal $\left(T_{e} \simeq T_{i}\right)$ plasma, owing to the interaction with background thermal ions. Thus, this mode may occur in a realistic magnetosphere only upon permanent driving, such as the considered pumping of external FMS wave energy into the resonant region. In this aspect, the SMS mode could be an intermediary, transferring energy from solar wind flow to external magnetospheric MHD disturbances, and eventually to localized plasma heating.

The comparison of field-aligned structure of the Pc5 event observed by Takahashi et al. (1988) with the predictions of our theoretical model shows that this scale matches well the results of numerical modeling of the field-aligned SMS oscillation structure (function $S_{4}$ ) of the harmonic with $N=4$. The polarization structure of the magnetic components in the magnetosonic resonance, $B_{z} \gg B_{x}>B_{y}$, corresponds to the class of compressional poloidal waves in the magnetosphere.

The drift instability mechanism can be operative chiefly during the main and recovery phases of magnetic storms when a sufficient amount of the ring current particles have been injected into the inner magnetosphere.

The SMS waves are to be strongly absorbed by resonant interaction with background thermal particles, while without energy pumping by an FMS mode they should soon die down. Thus, the resonant excitation of SMS oscillations can be an intermediary, transferring energy from the solar wind for the heating of the magnetospheric near-equatorial plasma.

\section{Conclusion}

In this paper we would like to draw attention to the possibility of resonant conversion of large-scale MHD disturbances into localized SMS oscillations. We have derived Eqs. (21) and (29), describing the structure and spectrum of resonant standing SMS waves in a dipole-like magnetosphere. We have also obtained the boundary conditions (19) for the resonant SMS waves at the ionosphere. Solutions have been found to equations describing the structure of resonant SMS oscillations, both along magnetic field lines, $S_{N}\left(x^{1}, x^{3}\right)$, and across magnetic shells, $U_{N}\left(x^{1}\right)$. Field-aligned structure has been examined both in the WKB approximation (23) and numerically (Fig. 5). It was shown that the amplitude of standing SMS waves decreases fast upon approaching the Earth's ionosphere. In this context, the conclusion has been made that the ionosphere cannot play any significant role either in the generation or dissipation of magnetospheric SMS waves. The SMS oscillations could be observed only near the magnetospheric equatorial plane.

Comparison of spatial structures and spectra of the Alfvén and SMS waves generated via the Alfvén and magnetosonic resonances (Figs. 3-6) has been made. The frequency spectra of the fundamental harmonics of standing Alfvén and SMS waves differ by about two orders of magnitude, so an effective coupling between these two branches of MHD oscillations is impossible in a mildly disturbed magnetosphere. The resonantly excited SMS oscillations can be an intermediary, transferring energy from the solar wind to the heating of the magnetospheric near-equatorial plasma.

Acknowledgements. This work was partially supported by grants 04-05-64321 and 06-05-64495 from the Russian Foundation for Basic Research, 05-1000008-7978 and 06-1000013-8922 from INTAS, and by Program of presidium of Russian Academy of Sciences \#16 and OFN RAS \#16. The useful comments of both referees are appreciated.

Topical Editor I. A. Daglis thanks two referees for their help in evaluating this paper.

\section{References}

Barfield, J. N. and McPherron, R. L.: Storm time Pc5 magnetic pulsations observed at synchronous orbit and their correlation with the partial ring current, J. Geophys. Res., 83, 739-743, 1978.

Chen, L. and Cowley, S. C.: On field line resonances of hydromagnetic Alfvén waves in a dipole magnetic field, Geophys. Res. Lett., 16, 895-897, 1989.

Chen, L. and Hasegawa, A.: A theory of long period magnetic pulsation. 1. Steady state excitation of field line resonances, J. Geophys. Res., 79, 1024-1032, 1974.

Cheng, C. Z. and Lin, C. S.: Eigenmode analysis of compressional waves in the magnetosphere, Geophys. Res. Lett., 14, 884-887, 1987.

Cheng, C. Z., Qian, Q., Takahashi, K., and Lui, A. T. Y. Ballooning-mirror instability and internally driven Pc 4-5 wave events, J. Geomag. Geoelectr., 46, 997-1006, 1994.

Chew, G. F., Goldberger, M. L., and Low, F. E.: The Boltzmann equation and the one-fluid hydromagnetic equations in the absence of particle collisions, Proc. Roy. Soc., A236, 112-118, 1956.

Greene, H. L., Engebretson, M. J., Erickson, K. N., Peterson, W. K., Trattner, K. H., Cheng, C. Z., and Russell, C. T.: Analysis of Compressional Pc 5 Magnetic Field Oscillations and Associated Energetic Ion Modulations Occurring on December 20, 2002 in the Dusk Sector Outer Magnetosphere, Chapman Conference on Magnetospheric ULF Waves, San Diego, 2005, Occurring on 20 December 2002 in the Dusk Sector Outer Magnetosphere, AGU Chapman Conference on Magnetospheric ULF Waves, San Diego, 57-58, 2005.

Glassmeier, K. H., Buchert, S., Motschmann, U., Korth, A., and Pedersen, A.: Concerning the generation of geomagnetic giant pulsations by drift-bounce resonance ring current instabilities, Ann. Geophys., 17, 338-350, 1999, http://www.ann-geophys.net/17/338/1999/.

Kivelson, M. G. and Southwood, D. J.: Coupling of global magnetospheric MHD eigenmodes to field line resonances, J. Geophys. Res., 91, 4345-4351, 1986.

Klimushkin, D. Yu.: Spatial structure of small-scale azimuthal hydrodynamic waves in an axisymmetric magnetospheric plasma with finite pressure, Plasma Physics Reports, 23, 858-871, 1997. 
Korn, G. A. and Korn, T. M.: Mathematical handbook for scientists and engineers, McGraw-Hill Book Company, 1968.

Krall, N. A. and Trivelpiece, A. W.: Principles of plasma physics, McGraw-Hill book company, 1973.

Leonovich, A. S.: A theory of field line resonance in a dipole-like axisymmetric magnetosphere, J. Geophys. Res., 106, 25803 $25812,2001$.

Leonovich, A. S. and Mazur, V. A.: Resonance excitation of standing Alfvén waves in an axisymmetric magnetosphere (monochromatic oscillations), Planet. Space Sci., 37, 10951108, 1989.

Leonovich, A. S. and Mazur, V. A.: A theory of transverse smallscale standing Alfvén waves in an axially symmetric magnetosphere, Planet. Space Sci., 41, 697-717, 1993.

Leonovich, A. S. and Mazur, V. A.: An electromagnetic field induced in the ionosphere and atmosphere by low-frequency Alfvén oscillations of the magnetosphere: general theory, Planet. Space Sci., 39, 529-546, 1991.

Leonovich, A. S. and Mazur, V. A.: Penetration to the Earth's surface of standing Alfvén waves excited by external currents in the ionosphere, Ann. Geophys., 14, 545-556, 1996, http://www.ann-geophys.net/14/545/1996/.

Leonovich, A. S. and Mazur, V. A.: A model equation for monochromatic standing Alfvén waves in the axially symmetric magnetosphere, J. Geophys. Res., 102, 11 443-11 456, 1997.

Leonovich, A. S. and Mazur, V. A.: Structure of magnetosonic eigenoscillations of an axisymmetric magnetosphere, J. Geophys. Res., 105, 27 707-27 716, 2000.

Leonovich, A. S., Mazur, V. A., and Cao, J. B.: Self-consistent model of a dipole-like mrgnetosphere with an azimuthal solar wind flow, J. Plasma Phys., 70, 99-111, 2004.

Lifshitz, A. E. and Fedorov, E. N.: Hydromagnetic oscillations of the magnetospheric resonator (in Russian), Doklady Akademii Nauk SSSR, 287, 90-94, 1986.

Nakamizo, A. and Iijima, T.: A new perspective on magnetotail disturbances in terms of inherent diamagnetic processes, J. Geophys. Res., 108(A7), 1286, doi:10.1029/2002JA009400, 2003.

Pilipenko, V. A.: ULF waves on the ground and in space, J. Atmos. Terr. Phys., 52, 1193-1209, 1990.

Pilipenko, V., Vellante, M., and Fedorov, E.: Distortion of the ULF wave spatial structure upon transmission through the ionosphere, J. Geophys. Res., 105, 21 225-21 236, 2000.

Pokhotelov, O. A., Pilipenko, V. A., Nezlina, Yu. M., Woch, J., Kremser, G., Korth, A., and Amata, E.: Excitation of high-beta plasma instabilities at the geostationary orbit: Theory and observations, Planet. Space Sci., 34, 695-712, 1986.
Radoski, H. R.: A theory of latitude dependent geomagnetic micropulsations: The asymptotic fields, J. Geophys. Res., 79, 595604, 1974.

Southwood, D. J.: Some features of field line resonances in the magnetosphere, Planet. Space Sci., 22, 483-492, 1974.

Southwood, D. J.: Local compressional hydromagnetic waves in the magnetospheric ring currents, Planet. Space Sci., 25, 549$554,1977$.

Southwood, D. J. and Saunders, M. A.: Curvature coupling of slow and Alfvén MHD waves in a magnetotail field configuration, Planet. Space Sci., 33, 127-134, 1985.

Southwood, D. J. and Kivelson, M. G.: The effect of parallel inhomogeneity of magnetospheric hydromagnetic wave coupling, J. Geophys. Res., 91, 6871-6877, 1986.

Takahashi, K., Fennell, J. F., Amata, E., and Higbie, P. R.: Fieldaligned structure of the stormtime Pc5 wave of November 14-15, 1979, J. Geophys. Res., 92, 5857-5864, 1987.

Tamao, T.: Transmission and coupling resonance of hydromagnetic disturbances in the non-uniform Earth's magnetosphere, Sci. Rep. Tohoku Univ., 5(17), 43-54, 1965.

Taylor, J. P. N. and Walker, A. D. M.: Theory of magnetospheric standing hydromagnetic waves with large azimuthal wave number. 2. Eigenmodes of the magnetosonic and Alfvén oscillations, J. Geophys. Res., 92, 10 046-10 052, 1987.

Walker, A. D. M.: Theory of magnetospheric standing hydromagnetic waves with large azimuthal wave number. 1. Coupled magnetosonic and Alfvén waves, J. Geophys. Res., 92, 10039 $10045,1987$.

Walker, A. D. M. and Pekrides, H.: Theory of magnetospheric standing hydromagnetic waves with large azimuthal wave number. 4. Standing waves in the ring current region, J. Geophys. Res., 101, 27 133-27 147, 1996.

Woch, J., Kremser, G., Korth, A., Pokhotelov, O. A., Pilipenko, V. A., Nezlina, Yu. M., and Amata, E.: Curvature-driven drift mirror instability in the magnetosphere, Planet. Space Sci., 36, 383-393, 1988.

Woch, J., Kremser, G., and Korth, A.: A comprehensive investigation of compressional ULF waves observed in the ring current, J. Geophys. Res., 95, 15 113-15 132, 1990.

Wright, A. N.: Coupling of fast and Alfvén modes in realistic magnetospheric geometries, J. Geophys. Res., 97, 6429-6438, 1992.

Yumoto, K.: Characteristics of localized resonance coupling oscillations of the slow magnetosonic wave in a non-uniform plasma, Planet. Space Sci., 33, 1029-1036, 1985. 